\title{
The impact of new assistive technologies on specific occupational risks for blind and visual impaired peoples
}

\author{
Doru Costin Darabont ${ }^{1 *}$, Daniel Onuț Badea ${ }^{1}$, Alina Trifu ${ }^{1}$, Paul Fogarassy ${ }^{2}$ \\ ${ }^{1}$ National Institute for Research and Development in Occupational Safety "Alexandru Darabont" \\ INCDPM, B-dul Ghencea 35A, Bucharest, Romania \\ ${ }^{2} \mathrm{SC}$ Baum Engineering, Traian Moşoiu 8 Street, Arad, Romania
}

\begin{abstract}
As employees, the blind and visual disabled peoples face specific occupational risks related to personal safety most of us are usually not aware. Many hazards, easy to avoid for sighted peoples, became extremely dangerous for peoples without visual perception. For example, an electric cable with discontinuous isolation is much easy to be avoided for sighted peoples, while for blind persons could be lethal, especially as they need to touch objects not only to manipulate them, but also to localize and recognize them. Beside orientation problems and obstacles avoidance, other less obvious threats exist. This paper presents the findings of an INCDPM study developed in collaborations with Baum Engineering that addresses the issues of using assistive materials and technologies for blind people at workplace. This assistive technologies, which are a most needed tool for the visually impaired people to access the labour market, could be also a danger if not properly installed and implemented. The most obvious risk for visual impaired persons using tactile floor, could be the slippery surfaces related accidents, if the products are not compliant. Another danger could be tactile materials for orientation; if their surface is damaged and sharp edges or corners are exposed. Such an accident could be of tremendous impact for a blind that need to rely continuously on his fingers for environment tactile exploration.
\end{abstract}

\section{Introduction}

Globally, according to the World Health Organization data, it is estimated that approximately 1.3 billion people live with some form of vision impairment. Of these, 39 million are blind and 246 million have a moderate or severe form of vision impairment [1]. In Romania, on December 31, 2018, there were 95,699 persons with visual disabilities of whom 50,555 were adults with severe disabilities. In Bucharest, the General Directorates for Child Assistance and Social Protection have registered 4,354 persons with visual impairments in the three degrees of disability (Table 1).

\footnotetext{
* Corresponding author: darabont d@yahoo.com
} 
Table 1. Disabled persons at country level, by types and degrees of deficiencies, on December 31, 2018

\begin{tabular}{|c|c|c|c|c|c|}
\hline \multirow{2}{*}{ Defienciecy } & \multirow{2}{*}{$\begin{array}{c}\text { Total } \\
\text { persons }\end{array}$} & \multicolumn{4}{|c|}{ Out of total, by degree of deficiencies } \\
\cline { 3 - 6 } & & Severe & Marked & Medium & Minor \\
\hline Auditive & 23.564 & 84 & 19.734 & 1.854 & 61 \\
\hline Visual & 95.699 & 50555 & 35830 & 5.983 & 605 \\
\hline Psihic & 91.683 & 21589 & 51906 & 3.189 & 242 \\
\hline $\begin{array}{c}\text { Deafness- } \\
\text { blindness }\end{array}$ & 89 & 12 & 63 & 3 & 0 \\
\hline
\end{tabular}

The blind and visual disabled peoples as employees face specific occupational risks related to personal safety most of us are usually not aware. Many hazards, easy to avoid for sighted peoples, became extremely dangerous for peoples without visual perception. For example, an electric cable with discontinuous isolation is much easy to be avoided for sighted peoples, while for blind persons could be lethal, especially as they need to touch objects not only to manipulate them, but also to localize and recognize them. Beside orientation problems and obstacles avoidance, other less obvious threats exist. This paper presents the findings of an INCDPM study developed in collaborations with Baum Engineering that addresses the issues of using assistive materials and technologies for blind people at workplace.

\section{Risk assessment methodology}

The methodology adopted for this paper consist in systematic searches that were made using the "Risk assessment files" database resources of National Research and Development Institute of Occupational Safety - INCDPM "Alexandru Darabont", on the most common professions that visually impaired occupied in Romania. Were targeted the occupations assessed by Institute' safety specialist in the last ten years.

Also a comprehensive review of the occupational safety literature was undertaken using the keywords: "occupational risks for disabled people", "assistive technologies for visually impaired people", on a range of online academic databases provided by: Science Direct, IEEE, PubMed, Web of Science and Google Scholar. In addition to the formal literature described above, a search of grey literature was conducted accessing a range of internet sites of national and international organisations recognised as having involvement in the discipline of occupational health and safety (OHS) or disability. Other than local sites, the primary focus for the project were the internet sites of disability management and research institutes in Europe, USA and Canada. From these principal sites, links to other resources were pursued as was appropriate. The following sources were specifically targeted: European Agency for Special Needs and Inclusive Education, National Institute of Disability Management and Research Canada, United States Department of Health and Human Services, Finnish Institute of Occupational Health, National Institute for Occupation Safety and Health USA, Health and Safety Executives. 


\section{Safety risks related to assistive technologies, devices and materials}

Assistive technology (AT) is considered to be all the systems, technologies, equipment, devices, apparatus, appliances, services, processes and environmental modifications, that are used by disabled people as help in their lives, make their activities easier, providing a safe mobility that enable them to overcome various physical, social, infrastructural and accessibility barriers to independence and live active, productive and independent lives as equal members of the society.

The current field of assistive technologies for blindness and visual impairment is quite complex and include haptic aids, travelling aids, AT for accessible information and communication, AT for daily living. Haptic aids are low-tech (e.g., white cane, traditional Braille system, embossed pictures, including tactile maps etc.) and high-tech, which includes advanced Braille applications, advanced canes, haptic aids for computer usage and matrices of point stimuli. The term 'haptic' or 'active touch' means "pertaining to the sense of touch", "tactile", which is a complex sensorial modality, covering systems that also use kinaesthetic information, generated by sensors in muscles, tendons and joints [2].

Visual assistive technology that became available for the blind people through electronic devices and use of function of sensors that also aid the user with the mobility task based on the determination of dimensions, range and height of the objects [3], can be divided into three categories: vision enhancement, vision substitution, and vision replacement $[4,5]$.

The vision replacement category is more complex than the other two categories because it deals with medical and technology issues and includes displaying information directly to the visual cortex of the brain or through an ocular nerve [4].

Aldo vision enhancement and vision substitution are similar in concept there is a difference between them: in vision enhancement, the camera input is processed and then the results will be visually displayed, meanwhile in vision substitution the result constitutes non-visual display, which can be vibration, auditory or both based on the hearing and touch senses that can be easily controlled and felt by the blind user.

The vision substitution category includes three subcategories: Electronic Travel Aid (ETAs) that are devices that gather information about the surrounding environment and transfer it to the user through sensor cameras, sonar, or laser scanners [6], Electronic Orientation Aid (EOAs) - devices that provide pedestrians with directions in unfamiliar places [7] and Position Locator Devices (PLDs) - devices that determine the precise position of its holder such as devices that use GPS technology.

ETAs are very useful in determining obstacles around the user body from the ground to the head, in affording some instructions to the user about the movement surface consists of gaps or textures, in finding items surrounding the obstacles, in providing information about the distance between the user and the obstacle with essential direction instructions, in proposing notable sight locations in addition to identification instructions and in affording information to give the ability of self-orientation and mental map of the surroundings. On the other hand, EOAs are used in defining the route to select the best path, in tracing the path to approximately calculate the location of the user and in providing mobility instructions and path signs to guide the user and develop her/his brain about the environment.

Some of assistive technologies are embedded in the environment, aimed at making it accessible and user-friendly and overcoming the main barriers met by disabled persons. Most of them are low-tech and include good lighting, well-designed signage systems, including tactile and audio information and alarms, tactile paving and surfaces to warn of hazards and to direct people, and colour contrasts, including colour-contrasting strips to make items easier to recognise. High-tech devices are electronic signal systems embedded 
in the environment which are activated by the presence of the disabled person. Talking signs are repeating, directionally selective voice messages transmitted by infrared light to a hand-held receiver.

Because all the AT mention above are either very expensive or in prototype state and very few of them are used on a daily basis at work, for the first phase of our research we approached and studied those assistive technologies that are already installed and used by visually impaired employees at a relatively large scale in our country: tactile floors and tactile materials for orientation.

\subsection{Tactile floors}

Designed to help the blind and visually impaired navigate both indoor and outdoor, tactile paving surfaces are used to convey important information to visually impaired pedestrians about their environment, for example, hazard warning, directional guidance, or the presence of an amenity. It is a known fact that visually impaired people can reliably detect, distinguish and remember a limited number of different tactile paving surfaces and the distinct meanings assigned to them.

There are two types of tactile ground surface indicators: warning indicators and directional indicators [8]. Warning indicators (a textured surface feature consisting of truncated domes built into or applied to walking surfaces) alert pedestrians to hazards in the continuous accessible path of travel, indicating that they should stop to determine the nature of the hazard before proceeding further, without indicate what the hazard will be (fig. 1).

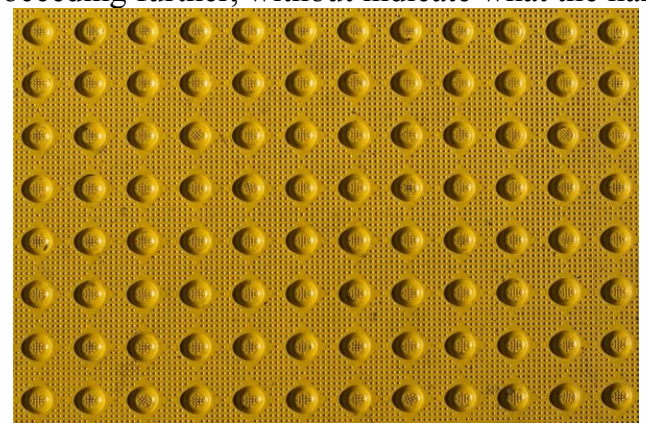

Fig. 1. Tactile paving - warning indicator

Directional indicators (a textured surface feature consisting of directional grooves built into or bars applied to walking surfaces) give directional orientation and designate the continuous accessible path of travel when other tactile or environmental cues are insufficient. When combined with other environmental information, tactile grounds surface indicators assist people who are blind or have low vision with their orientation and awareness of impending obstacles, hazards and changes in the direction of the continuous accessible path of travel (fig.2). 


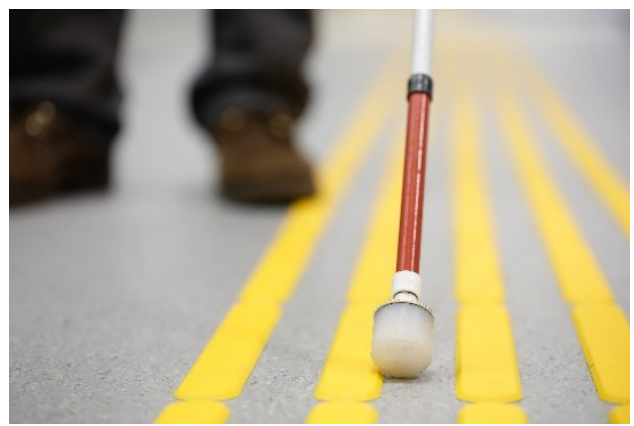

Fig. 2. Tactile paving - directional indicator

Warning indicators are installed to inform people who are blind or have low vision of the following hazards: life threatening hazards where serious falls may occur, such as at railway platforms or wharves, vehicle hazards on roads where the footpath is not separated from the roadway by an abrupt change of grade; approaches to stairways, ramps, escalators, railway crossings and moving walkways; vehicle hazards at busy vehicle crossing points including, but not limited to shopping centres, bus stations and large car parks; to designate the stopping point of the entry doors at bus stops.

Directional indicators give directional orientation to visually impaired but, as with any facility, directional indicators should be used appropriately and not overused. If overused, it can lead to pedestrians who are blind or have low vision being unable to tell the difference between indicators intended for different purposes. Also, directional indicators can sometimes be uncomfortable when being negotiated by wheelchair users, people using other ambulatory devices, prams etc. so other environmental solutions/indicators should be sought e.g. landscaping and visual contrast Directional indicators should only be used where other tactile and environmental cues, such as the property line or kerb edge, are either absent or give insufficient guidance.

Although tactile paving units are technically useful, being like Braille for pavements, there are cases in which these become a hazard. Installing the wrong pattern can give very misleading information to visually impaired people because each tactile pattern provides a specific message. Research has found that the majority of tactile surfaces are incorrectly installed [9]. Sometimes, the textured tiles have simply been misused as decorative touches, defeating their critical purpose (fig. 3). In others, angled tile patterns force blind lanes to zig and zag their way down a street, which would be confusing and frustrating to users.

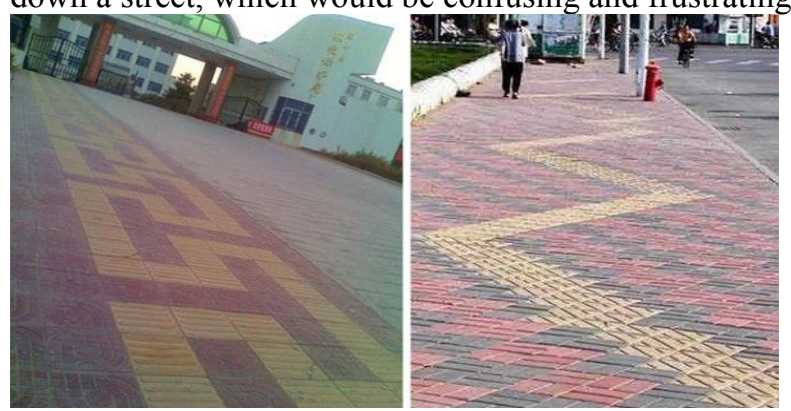

Fig. 3. Tactile paving used to create decorative patterns (left) or zigzag routes (right)

In the worst case scenario (fig. 4), paths leading into trees, holes, bars and even off bridgeside cliffs will became a source of potential injuries or fatalities. Tactile surfaces are designed to give a physical cue to a visually impaired individual to aid in navigating the local environment. If incorrectly installed, they can increase the risk of tripping. 

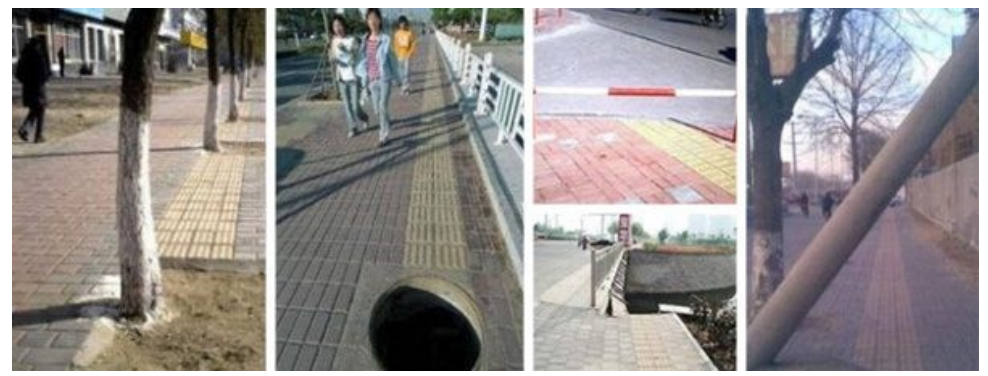

Fig. 4. "Worst case example" - paths leading into trees, holes, bars

Also there is a high risk of slipping if their wet. In almost all slip accidents there is some form of contamination that will reduce the amount of available friction between foot and floor. As with profiled surfaces, the raised pattern of a tactile surface will not necessarily provide enhanced slip resistance.

The materials selected for tactile paving must have slip resistance in wet and dry conditions, visual contrast, resistance to impact (chipping or cracking), shall exhibit weathering and UV stability for maintaining high visual colour contrast, wear resistance and adhesion/bond strength particularly if immersed in water [10].

The main requirements of the assistive device for blind person are safety, practicability, portability and convenience. Safety is the basic requirement to judge whether an assistive device is reliable or not. The most important task for the blind person is to gain information on the circumstances of the road and the location of obstacle.

\subsection{Tactile materials for orientation}

The sense of touch is one of the most important for blind people. Contrary popular beliefs a loss of sight is not accompanied by an increase in the effectiveness of other non-visual senses. However, it is generally accepted the fact that people who are blind or have low vision generally place more emphasis on information received via other senses, especially the sense of touch.

The most used tactile materials for orientation are Braille tactile indicators, tactile information panels as well as tactile maps (fig. 5). These materials must have a series of features that prevent any visually disabled people to suffer a cut or a deep wound because such an accident could be of tremendous impact for a blind that need to rely continuously on his fingers for environment tactile exploration. Their surface must not be damaged or have sharp edges or corners that are exposed.
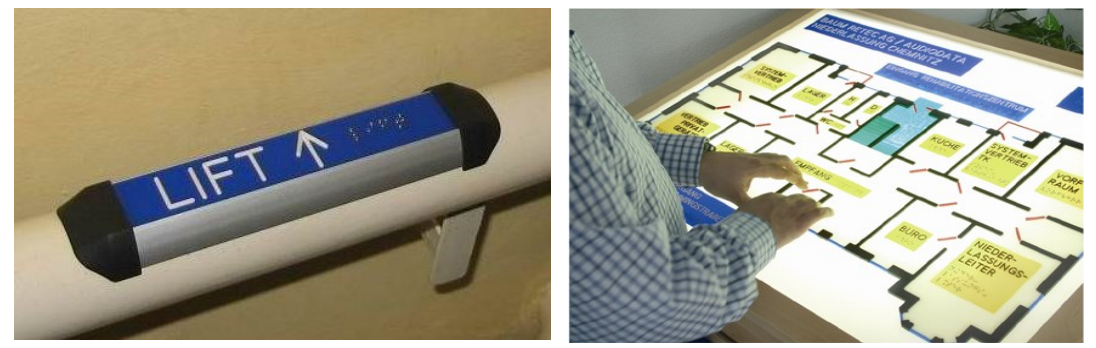

Fig. 5. Tactile materials for orientation 
If their surface is damaged and sharp edges or corners are exposed, such an accident could be of tremendous impact for a blind that need to rely continuously on his fingers for environment tactile exploration.

\section{Conclusions and further research}

The assistive technologies, which are a most needed tool for the visually impaired people to access the labour market, could be also a danger if not properly installed and implemented. The most obvious risk for visual impaired persons using tactile floor, could be the slippery surfaces related accidents, if the products are not compliant. Another hazard could be tactile materials for orientation if their surface is damaged and sharp edges or corners are exposed. Such an accident could be of tremendous impact for a blind that need to rely continuously on his fingers for environment tactile exploration. Further research will be performed in order to better investigate the specific risk for visual impaired peoples using other type of assistive technology.

Acknowledgement. This research was partially supported by a project co-financed from the European Regional Development Fund through the Competitiveness Operational Program 2014-2020, Priority Axis 1: Research, Technological Development and Innovation (RDI) in support of economic competitiveness and business development. Project title: Partnership for knowledge transfer and research development related to the assessment and prevention of occupational risks that can lead to disasters (PROC). ID/Cod SMIS 2014+: POC P_40_182/111954

\section{References}

1. World Health Organization. Visual Impairment and Blindness. Available online: at https://www.who.int/news-room/fact-sheets/detail/blindness-and-visual-impairment (accessed on 14 July 2019)

2. A. Bhowmick, S.M. Hazarika. An insight into assistive technology for the visually impaired and blind people: state-of-the-art and future trends. J Multimodal User Interfaces, 11, 149-172 (2017)

3. R. Tapu, B. Mocanu, A survey on wearable devices used to assist the visual impaired user navigation in outdoor environments. Proceedings of the 2014 11th International Symposium on Electronics and Telecommunications (ISETC), Timisoara, Romania, 14-15 November, 2014

4. D. Dakopoulos,; N.G. Bourbakis, Wearable obstacle avoidance electronic travel aids for blind: A survey. IEEE Trans. Syst. Man Cybern. Part C, 40, 25-35, (2010)

5. L. Renier, A.G. De Volder, Vision substitution and depth perception: Early blind subjects experience visual perspective through their ears. Disabil. Rehabil. Assist. Technol. 5, 175-183, (2010)

6. J. Liu, L. Xu, W. Jin. Electronic travel aids for the blind based on sensory substitution. Proceedings of the 2010 5th International Conference on Computer Science and Education (ICCSE), Hefei, China, 24-27 August (2010)

7. S. Kammoun, J.-M. Marc, B. Oriola, J. Christophe, Toward a better guidance in wearable electronic orientation aids. In Proceedings of the IFIP Conference on Human-Computer Interaction, Lisbon, Portugal, 5-9 September (2011)

8. Department of the Environment, Transport and the Regions, Guidance on the use of Tactile Paving Surfaces (Crown London Limited, 1998)

9. M.L. Morrey, Tactile Paving Survey, (Crown London Limited, 2005)

10. Guidelines for facilities for blind and vision impaired pedestrians, 2015, Available online at https://www.nzta.govt.nz/assets/resources/road-traffic-standards/docs/rts-14.pdf (accessed on 14 July 2019) 